\title{
Global existence and propagation speed for a generalized Camassa-Holm model with both dissipation and dispersion
}

\author{
Qiaoyi $\mathrm{Hu}^{1,2 *}$, Zhijun $\mathrm{Qiao}^{2 \dagger}$ \\ ${ }^{1}$ Department of Mathematics, South China Agricultural University, \\ Guangzhou, Guangdong 510642, PR China \\ ${ }^{2}$ School of Mathematical and Statistical Science, \\ University of Texas - Rio Grande Valley, \\ Edinburg, TX 78539, USA
}

\begin{abstract}
In this paper, we study a generalized Camassa-Holm $(\mathrm{gCH})$ model with both dissipation and dispersion, which has $(\mathrm{N}+1)$-order nonlinearities and includes the following three integrable equations: the Camassa-Holm, the Degasperis-Procesi, and the Novikov equations, as its reductions. We first present the local well-posedness and a precise blow-up scenario of the Cauchy problem for the $\mathrm{gCH}$ equation. Then we provide several sufficient conditions that guarantee the global existence of the strong solutions to the $\mathrm{gCH}$ equation. Finally, we investigate the propagation speed for the $\mathrm{gCH}$ equation when the initial data is compactly supported.
\end{abstract}

2000 Mathematics Subject Classification: 35G25, 35L05

Keywords: generalized Camassa-Holm $(\mathrm{gCH})$ model, Local wellposedness, Blow-up, Global solution, Propagation speed.

\section{Introduction}

Recently, Himonas and Holliman [25] studied the following generalized CamassaHolm equation

$$
u_{t}-u_{t x x}-u^{N} u_{x x x}-\beta u^{N-1} u_{x} u_{x x}+(\beta+1) u^{N} u_{x}=0
$$

where $N \in \mathbb{N}, \beta \in \mathbb{R}$, and proved the local well-posedness and the nonuniform dependence of its Cauchy problem in Sobolev spaces $H^{s}$ with $s>\frac{3}{2}$.

\footnotetext{
*e-mail: huqiaoyi@scau.edu.cn

${ }^{\dagger}$ e-mail: zhijun.qiao@utrgv.edu
} 
Zhou and $\mathrm{Mu}$ studied the persistence properties of strong solutions and the existence of its weak solutions to Eq. (1.1) [53]. Himonas and Thompson [29] also showed the persistence properties and unique continuation of Eq. (1.1). Eq. (1.1) is an evolution equation with $(N+1)$-order nonlinearities and includes three remarkable integrable equations: the Camassa-Holm $(\mathrm{CH})$ equation, the Degasperi-Procesi (DP) equation, and the Novikov equation (NE).

As $N=1$ and $\beta=2$, Eq.(1.1) reads as the well-known CamassaHolm equation, which models the unidirectional propagation of shallow water waves over a flat bottom, and is completely integrable with a biHamiltonian structure [2, 23]. The remarkable feature of the $\mathrm{CH}$ equation is its peaked soliton (peakon) solution in the form of $u(t, x)=c e^{-|x-c t|}$, where $c$ is a wave speed 2. The Cauchy problem of the $\mathrm{CH}$ equation has extensively been studied. For instance, its local well-posedness problem for initial data $u_{0} \in H^{s}$ with $s>\frac{3}{2}$ was studied in [6, 12, 33, 43]. More interestingly, the $\mathrm{CH}$ equation has not only global strong solutions modelling permanent waves [8, 12] but also blow-up solutions modelling wave breaking [7, 11, 12, 13, 33, 43]. On the other hand, the $\mathrm{CH}$ equation has globally weak solutions with initial data $u_{0} \in H^{1}[1,15,50]$ as well as the algebro-geometric solution [40].

As $N=1$ and $\beta=3$, Eq. (1.1) is cast to the Degasperi-Procesi (DP) equation [16]. The DP equation is another integrable peakon model with quadratic nonlinearity, but with $3 \times 3$ Lax pairs [17]. The well-posedness, global existence, and blow-up phenomena of DP equation may be seen in [4, 20, 21, 26, 27, 34, 51, 52, and the DP equation may also be generalized to an entire integrable hierarchy including both positive and negative flows [41.

As $N=2$ and $\beta=3$, Eq. (1.1) is reduced to the Novikov equation (NE) [36], which is also integrable with $3 \times 3$ Lax pairs and with the peakon solution $u(t, x)=\sqrt{c} e^{-|x-c t|}$ as well [30]. In [36], some integrable equations with cubic nonlinearities were produced including the NE and the cubic $\mathrm{CH}$ or FORQ equation [22, 38, 42]. The local well-posedness of the NE's Cauchy problem on both the line and the circle, and its global solution existence and global weak solutions were investigated in [28, 45, 48, 49].

In this paper, we study the Cauchy problem of the generalized CamassaHolm equation (1.1) with both arbitrary dissipation and dispersion

$$
\begin{cases}u_{t}-u_{t x x}-u^{N} u_{x x x}-\beta u^{N-1} u_{x} u_{x x}+(\beta+1) u^{N} u_{x} & \\ +k\left(1-\partial_{x}^{2}\right) u_{x}+\lambda\left(1-\partial_{x}^{2}\right) u=0, & t>0, x \in \mathbb{R}, \\ u(0, x)=u_{0}(x), & x \in \mathbb{R},\end{cases}
$$

where supp $u_{0} \subset[a, b]$ is a compactly supported initial data, $k \in \mathbb{R}$ is an arbitrary dispersion coefficient, and $\lambda>0$ is a dissipative parameter. A 
physical motivation of studying dissipative equations is to include energy dissipation mechanisms in the real world, which indeed occurs in our daily life. In the literature there were many articles dealing with nonlinear models with dissipation. Ott and Sudan [39] investigated how the KdV equation was modified by the presence of dissipation and what the effect of such a dissipation was on soliton solutions of the KdV equation. Ghidaglia 24] studied the long time behavior of solutions to the weakly dissipative $\mathrm{KdV}$ equation as a finite dimensional dynamical system. Wu and Yin discussed the blow-up, and blow-up rate and decay of solutions to the weakly dissipative periodic $\mathrm{CH}$ equation (i.e. Eq.(1.2) with $N=1, \beta=2, k=0$ ) [46]. Thereafter, they also investigated the blow-up and decay of solutions to the weakly dissipative DP equation (i.e. Eq.(1.2) with $N=1, \beta=3, k=0$ ) on the line [47. Hu and Yin discussed the blow-up and blow-up rate of solutions to a weakly dissipative periodic rod equation [18]. Later, Hu studied the global existence and blow-up phenomena for a weakly dissipative periodic 2-component Camassa-Holm system [19]. In 2014, Zhou, Mu and Wang [54] considered the weakly dissipative gCH equation (i.e. Eq.(1.2) with $k=0$ ). However, the work done above was only involved in dissipative terms. Recently, Novruzov and Hagverdiyev [37] analyzed the behavior of solutions to the dissipative $\mathrm{CH}$ equation with arbitrary dispersion coefficient (i.e. Eq.(1.2) with $N=1, \beta=2$ ).

In this paper, we discuss the local well-posedness, the global existence, and the propagation speed of strong solutions to Eq. (1.2). Our study indicates that in comparison between $\mathrm{Eq}(1.1)(k=\lambda=0)$ and Eq. (1.2) $(k, \lambda \neq 0)$, some behaviors of solutions to the gCH equation (1.2) with dissipation and dispersion are similar to the ones of Eq. (1.1), such as, the local well-posedness and the blow-up scenario. However, the dissipative term $\lambda\left(u-u_{x x}\right)$ and the dispersive term $k\left(u_{x}-u_{x x x}\right)$ in Eq. (1.2) do have impacts on the global existence and the propagation speed of its solutions, which are shown below in Theorem 3.1 and Theorem 4.1, respectively. In particular, the propagation speed is seriously affected by both the dissipative parameter $\lambda$ and the dispersion coefficient $k$. On the other hand, it is worthy to note that the main difficulty in establishing the above results lies in controlling certain norms of $(N+1)$-order nonlinearities. In addition, some of our results cover the earlier corresponding results studied in [29, 37].

The paper is organized as follows. In the second section we give some preliminary results including the local well-posedness of Eq.(1.2), the precise blowup scenario and some useful lemmas to study the global existence and the propagation speed. In the third section we provide several global existence results, which reveal that Eq.(1.2) has global solutions modelling permanent waves. In the fourth section we study the propagation speed of strong solutions to Eq.(1.2) under the condition that the initial data has compact support. 
Notation. Throughout the paper, $*$ is referred to the convolution. The norm in the Lebesgue space $L^{p}(\mathbb{R})$ is denoted by $\|\cdot\|_{L^{p}}$, while $\|\cdot\|_{H^{s}}, s \in \mathbb{R}$, stands for the norm in the classical Sobolev spaces $H^{s}(\mathbb{R})$, where $1 \leq p \leq \infty$.

\section{Preliminaries}

In this section, we display some necessary results in order to reach our goal. Let us first present the local well-posedness for the Cauchy problem of Eq.(1.2) in $H^{s}(\mathbb{R}), s>\frac{3}{2}$. Hence, we rewrite Eq.(1.2) in the form of a quasi-linear evolution equation of hyperbolic type. Letting $y:=u-u_{x x}$ yields

$$
\begin{cases}y_{t}+y_{x}\left(u^{N}+k\right)+\frac{\beta}{N} y\left(u^{N}\right)_{x}+\lambda y & =0, \\ y(0, x)=u_{0}(x)-\partial_{x}^{2} u_{0}(x), & t>0, x \in \mathbb{R}, \\ & x \in \mathbb{R} .\end{cases}
$$

Note that $G(x):=\frac{1}{2} e^{-|x|}$ is the kernel of $\left(1-\partial_{x}^{2}\right)^{-1}$. Then $\left(1-\partial_{x}^{2}\right)^{-1} f=G * f$ for all $f \in L^{2}(\mathbb{R})$ and $G * y=u$. Therefore, Eq.(2.1) can be reformulated in the following form:

$$
\begin{cases}u_{t}+\left(u^{N}+k\right) u_{x}+\partial_{x} G * h+G * g=0, & \\ u(0, x)=u_{0}(x), & t>0, x \in \mathbb{R},\end{cases}
$$

or an equivalent form:

$$
\begin{cases}u_{t}+\left(u^{N}+k\right) u_{x}=-\partial_{x}\left(1-\partial_{x}^{2}\right)^{-1} h-\left(1-\partial_{x}^{2}\right)^{-1} g, & t>0, x \in \mathbb{R} \\ u(0, x)=u_{0}(x), & x \in \mathbb{R}\end{cases}
$$

where

$$
h:=\frac{\beta}{N+1} u^{N+1}+\frac{3 N-\beta}{2} u^{N-1} u_{x}^{2}-\lambda u_{x}
$$

and

$$
g:=\frac{(N-1)(\beta-N)}{2} u^{N-2} u_{x}^{3}+\lambda u .
$$

Note that, the local well-posedness of the Cauchy problem for Eq. (1.2) (or Eq.(2.3)) in $H^{s}(\mathbb{R}), s>\frac{3}{2}$ with $\lambda=k=0$ can be obtained by Kato's semigroup theorem [31] (see, for example, [53]), or by applying the contractionmapping principle (see, [25]).

Theorem 2.1. Given $u_{0} \in H^{s}(\mathbb{R}), s>\frac{3}{2}$, there exists a maximal $T>0$, and a unique solution u to Eq.(1.2) (or Eq.(2.3)) such that

$$
u=u\left(., u_{0}\right) \in C\left([0, T) ; H^{s}(\mathbb{R})\right) \cap C^{1}\left([0, T) ; H^{s-1}(\mathbb{R})\right) .
$$

Moreover, the solution depends continuously on the initial data, i.e. the mapping $u_{0} \rightarrow u\left(., u_{0}\right): H^{s}(\mathbb{R}) \rightarrow C\left([0, T) ; H^{s}(\mathbb{R})\right) \cap C^{1}\left([0, T) ; H^{s-1}(\mathbb{R})\right)$ is 
continuous. Furthermore, $T$ may be chosen independent of $s$ in the following sense. If $u \in C\left([0, T) ; H^{s}(\mathbb{R})\right) \cap C^{1}\left([0, T) ; H^{s-1}(\mathbb{R})\right)$ is a solution to Eq.(1.2), and if $u_{0} \in H^{s^{\prime}}(\mathbb{R})$ for some $s^{\prime} \neq s, s^{\prime}>\frac{3}{2}$, then

$$
u \in C\left([0, T) ; H^{s^{\prime}}(\mathbb{R})\right) \cap C^{1}\left([0, T) ; H^{s-1}(\mathbb{R})\right) .
$$

In particular, if $u_{0} \in C^{\infty}(\mathbb{R})=\bigcap_{s \geq 0} H^{s}(\mathbb{R})$, then $u \in C\left([0, T) ; C^{\infty}(\mathbb{R})\right)$.

Next, we address the global existence of Eq. (1.2). To see this, let us recall the following useful lemmas.

Lemma 2.1. [32] If $r>0$, then $H^{r} \cap L^{\infty}$ is an algebra. Moreover

$$
\|f g\|_{H^{r}} \leq c\left(\|f\|_{L^{\infty}}\|g\|_{H^{r}}+\|f\|_{H^{r}}\|g\|_{L^{\infty}}\right),
$$

where $c$ is a constant depending only on $r$.

Lemma 2.2. [32] If $r>0$, then

$$
\left\|\left[\Lambda^{r}, f\right] g\right\|_{L^{2}} \leq c\left(\left\|f_{x}\right\|_{L^{\infty}}\left\|\Lambda^{r-1} g\right\|_{L^{2}}+\left\|\Lambda^{r} f\right\|_{L^{2}}\|g\|_{L^{\infty}}\right),
$$

where $c$ is a constant depending only on $r$.

Lemma 2.3. [15] Let $g \in C^{m+2}(\mathbb{R})$ and $g(0)=0$. Then for every $\frac{1}{2}<r \leq$ $m$, we have

$$
\|g(u)\|_{r} \leq \tilde{g}\left(\|u\|_{L^{\infty}}\right)\|u\|_{r}, \quad u \in H^{r} .
$$

where $\tilde{g}$ is a monotone increasing function depending only on the $g$ and $r$.

Lemma 2.4. [32] Let $f \in H^{s}, s>\frac{3}{2}$. Then

$$
\left\|\Lambda^{-r}\left[\Lambda^{r+t+1}, M_{f}\right] \Lambda^{-t}\right\|_{L^{2}} \leq c\|f\|_{H^{s}}, \quad|r|,|t| \leq s-1,
$$

where $M_{f}$ is the operator of multiplication by $f$ and $c$ is a positive constant depending only on $r, t$.

Lemma 2.5. [31] Let $r, t$ be real numbers such that $-r<t \leq r$. Then

$$
\begin{gathered}
\|f g\|_{H^{t}} \leq c\|f\|_{H^{r}}\|g\|_{H^{t}}, \quad \text { if } r>\frac{1}{2}, \\
\|f g\|_{H^{t+r-\frac{1}{2}}} \leq c\|f\|_{H^{r}}\|g\|_{H^{t}}, \text { if } r<\frac{1}{2},
\end{gathered}
$$

where $c$ is a positive constant depending on $r$, $t$.

Let us now give the global existence result. 
Theorem 2.2. Let $u_{0} \in H^{s}, s>\frac{3}{2}$, and let $T$ be the maximal existence time of the solution u to Eq.(1.2)(or Eq. (2.3)) with the initial data $u_{0}$. If there exists $M>0$ such that

$$
\|u(t, \cdot)\|_{L^{\infty}}+\left\|u_{x}(t, \cdot)\right\|_{L^{\infty}} \leq M, \quad t \in[0, T),
$$

then the $H^{s}-$ norm of the solution $u$ does not blow up in finite time.

Proof. Let $u$ be the solution to Eq. (2.3) with the initial data $u_{0} \in H^{s}, s>\frac{3}{2}$, and let $T$ be the maximal existence time of the corresponding solution $u$, which is guaranteed by Theorem 2.1.

Applying the operator $\Lambda^{s}$ to Eq. (2.3), multiplying by $\Lambda^{s} u$, and integrating over $\mathbb{R}$, we obtain

$$
\begin{aligned}
\frac{d}{d t}\|u\|_{s}^{2} & =-2\left(\left(u^{N}+k\right) u_{x}, u\right)_{s}+2\left(u, f_{11}\right)_{s}+2\left(u, f_{12}\right)_{s} \\
& :=I+I I+I I I
\end{aligned}
$$

where

$$
f_{11}=-\Lambda^{-2} g, \quad f_{12}=-\partial_{x} \Lambda^{-2} h .
$$

Let us estimate $I, I I$ and $I I I$. Noting $\left(\Lambda^{s} u_{x}, \Lambda^{s} u\right)_{L^{2}}=0$, we have

$$
\begin{aligned}
|I|= & \left|-2\left(\left(u^{N}+k\right) u_{x}, u\right)_{s}\right|=2\left|\left(\Lambda^{s}\left(u^{N} u_{x}\right), \Lambda^{s} u\right)_{L^{2}}\right| \\
= & 2\left|\left(\left[\Lambda^{s}, u^{N}\right] \partial_{x} u, \Lambda^{s} u\right)_{L^{2}}+\left(u^{N} \Lambda^{s} \partial_{x} u, \Lambda^{s} u\right)_{L^{2}}\right| \\
\leq & 2\left\|\left[\Lambda^{s}, u^{N}\right] \partial_{x} u\right\|_{L^{2}}\left\|\Lambda^{s} u\right\|_{L^{2}}+\left|\left(\left(u^{N}\right)_{x} \Lambda^{s} u, \Lambda^{s} u\right)_{L^{2}}\right| \\
\leq & c\left(\left\|\left(u^{N}\right)_{x}\right\|_{L^{\infty}}\|\| \Lambda^{s-1} u_{x}\left\|_{L^{2}}+\right\| \Lambda^{s} u^{N}\left\|_{L^{2}}\right\| u_{x} \|_{L^{\infty}}\right)\left\|\Lambda^{s} u\right\|_{L^{2}} \\
& +\left\|\left(u^{N}\right)_{x}\right\|_{L^{\infty}}\left|\left(\Lambda^{s} u, \Lambda^{s} u\right)_{L^{2}}\right| \\
\leq & c\left(\left\|N u^{N-1} u_{x}\right\|_{L^{\infty}}\|u\|_{H^{s}}+\tilde{g_{1}}\left(\|u\|_{L^{\infty}}\right)\|u\|_{H^{s}}\left\|u_{x}\right\|_{L^{\infty}}\right)\|u\|_{H^{s}}+\left\|\left(u^{N}\right)_{x}\right\|_{L^{\infty}} \mid\|u\|_{H^{s}}^{2} \\
\leq & c\left(2 N M^{N}+M \tilde{g_{1}}(M)\right)\|u\|_{H^{s}}^{2}:=C \|_{H^{s}},
\end{aligned}
$$

where we use Lemma 2.2 with $r=s, f=u^{N}, g=u_{x}$ when estimating $\left\|\left[\Lambda^{s}, u^{N}\right] \partial_{x} u\right\|_{L^{2}}$ and Lemma 2.3 with $r=s, g_{1}(u)=u^{N}$ when estimating $\left\|\Lambda^{s} u^{N}\right\|_{L^{2}}$.

Obviously, when $N=1,|I I|$ is easy to get. When $N=2$, we refer to the proof of Theorem 3.1 in [48. Without loss of generality, we assume that 
$N \geq 3$

$$
\begin{aligned}
|I I|= & \left|2\left(f_{11}(u), u\right)_{s}\right|=2\left|\left(\Lambda^{s-2}\left(\frac{(N-1)(\beta-N)}{2} u^{N-2} u_{x}^{3}+\lambda u\right), \Lambda^{s} u\right)_{L^{2}}\right| \\
\leq & 2 \mid\left(\left[\Lambda^{s-1}\left(\frac{(N-1)(\beta-N)}{2} u^{N-2} u_{x}^{3}+\lambda u\right), \Lambda^{s-1} u\right)_{L^{2}} \mid\right. \\
\leq & c\left(\left|\left(\left[\Lambda^{s-1}, u^{N-2}\right] u_{x}^{3}, \Lambda^{s-1} u\right)_{L^{2}}+\left(u^{N-2} \Lambda^{s-1} u_{x}^{3}, \Lambda^{s-1} u\right)_{L^{2}}\right|+\left\|\Lambda^{s-1} u\right\|_{L^{2}}^{2}\right) \\
\leq & c\left(\left\|\left[\Lambda^{s-1}, u^{N-2}\right] u_{x}^{3}\right\|_{L^{2}}\left\|\Lambda^{s-1} u\right\|_{L^{2}}+\left\|u^{N-2} \Lambda^{s-1} u_{x}^{3}\right\|_{L^{2}}\left\|\Lambda^{s-1} u\right\|_{L^{2}}+\|u\|_{H^{s-1}}^{2}\right) \\
\leq & c\left(\left\|\left(u^{N-2}\right)_{x}\right\|_{L^{\infty}}\left\|u_{x}^{3}\right\|_{H^{s-1}}+\left\|u_{x}^{3}\right\|_{L^{\infty}}\left\|u^{N-2}\right\|_{H^{s-1}}\right)\|u\|_{H^{s-1}} \\
& +c\left(\left\|u^{N-2}\right\|_{L^{\infty}}\left\|u_{x}^{3}\right\|_{H^{s-1}}\|u\|_{H^{s-1}}+\|u\|_{H^{s-1}}^{2}\right) \\
\leq & c\left[\left\|(N-2) u^{N-3} u_{x}\right\|_{L^{\infty}} \tilde{g_{2}}\left(\left\|u_{x}\right\|_{L^{\infty}}\right)\left\|u_{x}\right\|_{H^{s-1}}+\left\|u_{x}\right\|_{L^{\infty}}^{3}\left\|\tilde{g_{3}}\left(\|u\|_{L^{\infty}}\right)\right\| u \|_{H^{s-1}}\right]\|u\|_{H^{s-1}} \\
& +c\left[\left\|u^{N-2}\right\|_{L^{\infty}} \tilde{g_{2}}\left(\left\|u_{x}\right\|_{L^{\infty}}\right)\left\|u_{x}\right\|_{H^{s-1}}\|u\|_{H^{s-1}}+\|u\|_{H^{s-1}}^{2}\right] \\
\leq & c\left((N-2) M^{N-2} \tilde{g_{2}}(M)+M^{3} \tilde{g}_{3}(M)+M^{N-2} \tilde{g_{2}}(M)+1\right)\|u\|_{H^{s}}^{2} \\
:= & C\|u\|_{H^{s}}^{2},
\end{aligned}
$$

where we use Lemma 2.2 with $r=s-1, f=u^{N-2}, g=u_{x}^{3}$ when estimating $\left\|\left[\Lambda^{s-1}, u^{N-2}\right] u_{x}^{3}\right\|_{L^{2}}$ and Lemma 2.3 with $r=s-1, g_{2}\left(u_{x}\right)=u_{x}^{3}$ and $g_{3}(u)=$ $u^{N-2}$ when estimating $\left\|u_{x}^{3}\right\|_{H^{s-1}}$ and $\left\|u^{N-2}\right\|_{H^{s-1}}$.

Note that $H^{s}$ and $H^{s-1}$ are algebraic with $s>\frac{3}{2}$. Hence

$$
\begin{aligned}
|I I I| & =\left|2\left(f_{12}(u), u\right)_{s}\right| \leq 2\left\|f_{12}(u)\right\|_{H^{s}}\|u\|_{H^{s}} \\
\leq & \left\|\frac{\beta}{N+1} u^{N+1}+\frac{3 N-\beta}{2} u^{N-1} u_{x}^{2}-\lambda u_{x}\right\|_{H^{s-1}}\|u\|_{H^{s}} \\
\leq & c\|u\|_{H^{s}}\left(\left\|u^{N+1}\right\|_{H^{s-1}}+\left\|u^{N-1} u_{x}^{2}\right\|_{H^{s-1}}+\left\|u_{x}\right\|_{H^{s-1}}\right) \\
\leq & c\|u\|_{H^{s}}\left(\tilde{g_{4}}\left(\|u\|_{L^{\infty}}\right)\|u\|_{H^{s-1}}+\left\|u_{x}^{2}\right\|_{L^{\infty}}\left\|u^{N-1}\right\|_{H^{s}}+\left\|u_{x}^{2}\right\|_{H^{s-1}}\left\|u^{N-1}\right\|_{L^{\infty}}+\|u\|_{H^{s}}\right) \\
\leq & c\|u\|_{s}\left[\tilde{g_{4}}\left(\|u\|_{L^{\infty}}\right)\|u\|_{H^{s-1}}+\left\|u_{x}^{2}\right\|_{L^{\infty}} \tilde{g_{5}}\left(\|u\|_{L^{\infty}}\right)\|u\|_{H^{s}}\right. \\
& \left.+\tilde{g_{6}}\left(\left\|u_{x}\right\|_{L^{\infty}}\right)\left\|u_{x}\right\|_{H^{s-1}}\left\|u^{N-1}\right\|_{L^{\infty}}+\|u\|_{H^{s}}\right] \\
\leq & c\left(\tilde{g_{4}}(M)+M^{2} \tilde{g_{5}}(M)+M^{N-1} \tilde{g_{6}}(M)\right)\|u\|_{H^{s}}^{2} \\
:= & C\|u\|_{H^{s}}^{2},
\end{aligned}
$$

where we use Lemma 2.1 with $r=s-1$ when estimating $\left\|u^{N-1} u_{x}^{2}\right\|_{s-1}$ and Lemma 2.3 with $r=s-1, g_{4}=u^{N+1}, g_{5}=u^{N-1}, g_{6}\left(u_{x}\right)=u_{x}^{2}$, when estimating $\left\|u^{N+1}\right\|_{H^{s-1}},\left\|u^{N-1}\right\|_{H^{s-1}}$ and $\left\|u_{x}^{2}\right\|_{H^{s-1}}$.

Therefore

$$
\frac{d}{d t}\|u\|_{H^{s}}^{2} \leq C\|u\|_{H^{s}}^{2} .
$$

Application of the Gronwall's inequality and assumption of the theorem lead to

$$
\|u\|_{H^{s}}^{2} \leq \exp (C t)\left\|u_{0}\right\|_{H^{s}}^{2},
$$

which completes the proof of the theorem. 
Now, we come to present the precise blow-up scenario.

Theorem 2.3. If $u_{0} \in H^{s}(\mathbb{R}), s>\frac{3}{2}$, then the solution $u=u\left(., u_{0}\right)$ of Eq.(1.2) blows up in the finite time $T<+\infty$ if and only if

$$
\underset{t \uparrow T}{\limsup }\left\|u_{x}(t, \cdot)\right\|_{L^{\infty}}=+\infty \text { or } \limsup _{t \uparrow T}\|u(t, \cdot)\|_{L^{\infty}}=+\infty .
$$

Proof. Assume $u_{0} \in H^{s}$ for some $s \in N, s \geq 2$. Multiplying both sides of Eq.(2.1) by $y$ and using integration by parts with respect to $x$, we obtain

$$
\begin{aligned}
\int_{\mathbb{R}} 2 y y_{t} & =-2 \int_{\mathbb{R}} y_{x} u^{N} y d x-\frac{2 \beta}{N} \int_{\mathbb{R}} y^{2}\left(u^{N}\right)_{x} d x-2 k \int_{\mathbb{R}} y y_{x} d x-2 \lambda \int_{\mathbb{R}} y^{2} d x \\
& =\left(1-\frac{2 \beta}{N}\right) \int_{\mathbb{R}} y^{2}\left(u^{N}\right)_{x} d x-2 \lambda \int_{\mathbb{R}} y^{2} d x \\
& =\left(1-\frac{2 \beta}{N}\right) \int_{\mathbb{R}} y^{2} N u^{N-1} u_{x} d x-2 \lambda \int_{\mathbb{R}} y^{2} d x
\end{aligned}
$$

which implies the following result: if $\|u\|_{L^{\infty}}$ and $\left\|u_{x}\right\|_{L^{\infty}}$ are bounded on $[0, T)$, i.e. there exists a positive constant $M$ such that $\|u\|_{L^{\infty}}, \quad\left\|u_{x}\right\|_{L^{\infty}} \leq$ $M$, then we have

$$
\frac{d}{d t} \int_{\mathbb{R}} y^{2} d x \leq c\left(M^{N}+1\right) \int_{\mathbb{R}} y^{2} d x .
$$

Substituting $y=u-u_{x x}$ into the above inequality, noticing

$$
\|u\|_{H^{2}}^{2} \leq\|y\|_{L^{2}}^{2} \leq 2\|u\|_{H^{2}}^{2},
$$

and using the Gronwall's inequality, we arrive at

$$
\begin{aligned}
\|u\|_{H^{2}}^{2} & \leq \int_{\mathbb{R}} y^{2} d x \leq e^{c\left(M^{N}+1\right) T} \int_{\mathbb{R}} y_{0}^{2} d x \\
& \leq 2 e^{c\left(M^{N}+1\right) T}\left\|u_{0}\right\|_{H^{2}}^{2},
\end{aligned}
$$

which implies that the $H^{2}$-norm of the solution to Eq.(2.1) does not blow up in a finite time. By the Sobolev's embedding theorem, Theorem 2.2 ensures that $\|u\|_{H^{s}}$ does not blow up in a finite time.

On the other hand, by Sobolev's imbedding theorem, if

$$
\underset{t \uparrow T}{\limsup }\left\{\sup _{x \in \mathbb{R}}\left\|u_{x}(t, \cdot)\right\|_{L^{\infty}}\right\}=+\infty \text { or } \limsup _{t \uparrow T}\left\{\sup _{x \in \mathbb{R}}\|u(t, \cdot)\|_{L^{\infty}}\right\}=+\infty,
$$

then the solution will blow up in a finite time.

Applying Theorem 2.2 and a simple density argument, one may know that Theorem 2.3 holds for all $s>\frac{3}{2}$.

Remark 2.1. Theorem 2.3 covers Lemma 5.1 in [29]. 
Let us now give the following key lemmas which will be used later on in some theorems.

Lemma 2.6. Let $u_{0} \in H^{1}(\mathbb{R})$, and $\beta=N+1$, then as long as the solution $u(t)$ given by Theorem 2.1 exists for any $t \in[0, T)$, we have

$$
\|u\|_{H^{1}}^{2}=e^{-2 \lambda t}\left\|u_{0}\right\|_{H^{1}}^{2} .
$$

Proof. Multiplying both side of Eq.(2.1) by $u$ and integrating by parts, we obtain

$$
\int_{\mathbb{R}} u y_{t} d x+\int_{\mathbb{R}} u u^{N} y_{x} d x+k \int_{\mathbb{R}} u y_{x} d x+\frac{N+1}{N} \int_{\mathbb{R}} u\left(u^{N}\right)_{x} y d x+\int_{\mathbb{R}} \lambda y u d x=0 .
$$

Noticing

$$
\int_{\mathbb{R}} u^{N+1} y_{x} d x+\int_{\mathbb{R}} \frac{N+1}{N} \int_{\mathbb{R}} u\left(u^{N}\right)_{x} y d x=0 \quad \text { and } \quad k \int_{\mathbb{R}} u y_{x} d x=0,
$$

we have

$$
\frac{1}{2} \frac{d}{d t} \int_{\mathbb{R}}\left(u^{2}+u_{x}^{2}\right) d x+\lambda \int_{\mathbb{R}}\left(u^{2}+u_{x}^{2}\right) d x=0
$$

namely,

$$
\frac{d}{d t}\|u\|_{H^{1}}^{2}+2 \lambda\|u\|_{H^{1}}^{2}=0
$$

which implies the desired result in the lemma.

So, given initial data $u_{0} \in H^{s}(\mathbb{R}), s \geq 2$, Theorem 2.1 ensures the existence and uniqueness of strong solutions to Eq.(1.2). Let us consider the following initial value problem:

$$
\begin{cases}q_{t}(t, x)=u^{N}(t, q(t, x))+k, & t \in[0, T), x \in \mathbb{R} \\ q(0, x)=x, & x \in \mathbb{R}\end{cases}
$$

where $u$ denotes the solution to Eq.(1.2) with the initial data $u_{0}$. Because $u(t,.) \in H^{2}(\mathbb{S}) \subset C^{m}(\mathbb{S})$ with $0 \leq m \leq \frac{3}{2}, u \in C^{1}([0, T) \times \mathbb{R}, \mathbb{R})$. Applying classical results in the theory of ordinary differential equations may yield the following lemma, which is a key in the proof of global existence of solutions to Eq.(1.2) in Theorem 2.1.

Lemma 2.7. [35] Let $u_{0} \in H^{s}(\mathbb{R}), s \geq 2$, and let $T>0$ be the maximal existence time of the solution $u$ to Eq.(1.2) under the initial data $u_{0}$. Then Eq.(2.5) has a unique solution $q \in C^{1}([0, T) \times \mathbb{R}, \mathbb{R})$. Moreover, the map $q(t, \cdot)$ is an increasing diffeomorphism of $\mathbb{R}$ with the following positive derivative with respect to $x$ :

$$
q_{x}(t, x)=\exp \left(\int_{0}^{t}\left(N u^{N-1} u_{x}\right)(s, q(s, x)) d s\right)>0, \quad(t, x) \in[0, T) \times \mathbb{R} .
$$


Proof. Differentiating Eq. (2.5) with respect to $x$, we have

$$
\begin{cases}\frac{d}{d t} q_{x}(t, x)=N u^{N-1}(t, q(t, x)) q_{x}(t, x), & t \in[0, T), x \in \mathbb{R}, \\ q_{x}(0, x)=1, & x \in \mathbb{R},\end{cases}
$$

Solving the above equation leads to the desired result in the lemma.

Lemma 2.8. Let $u_{0} \in H^{s}(\mathbb{R}), s \geq 2$, and let $T>0$ be the maximal existence time of the solution $u$ to Eq.(1.2) corresponding to the initial data $u_{0}$. Then we have

$$
y(t, q(t, x)) q_{x}^{\frac{\beta}{N}}(t, x)=y_{0}(x) e^{-\lambda t}, \quad(t, x) \in[0, T) \times \mathbb{R},
$$

which implies

$$
e^{-\lambda t}\left\|y_{0}\right\|_{L^{\frac{N}{\beta}}(\mathbb{R})}=\|y\|_{L^{\frac{N}{\beta}}(\mathbb{R})} .
$$

In particular, if $N=2 \beta$, we have

$$
e^{-\lambda t}\left\|y_{0}\right\|_{L^{2}(\mathbb{R})}=\|y\|_{L^{2}(\mathbb{R})} .
$$

Proof. By Eq. (2.1), a direct calculation gives

$$
\begin{aligned}
& \frac{d}{d t}\left[y(t, q(t, x)) q_{x}^{\frac{\beta}{N}}\right]=\left(y_{t}+y_{x} q_{t}\right) q_{x}^{\frac{\beta}{N}}+\frac{\beta}{N} y q_{x}^{\frac{\beta}{N}-1} q_{t x} \\
= & {\left[y_{t}+y_{x}\left(u^{N}+k\right)\right] q_{x}^{\frac{\beta}{N}}+\frac{\beta}{N} y q_{x}^{\frac{\beta}{N}-1}\left(u^{N}\right)_{x} q_{x} } \\
= & \left(y_{t}+y_{x} u^{N}+k y_{x}+\frac{\beta}{N} y\left(u^{N}\right)_{x}\right) q_{x}^{\frac{\beta}{N}}=-\lambda y q_{x}^{\frac{\beta}{N}} .
\end{aligned}
$$

Solving for $y$ from the above equation, we obtain

$$
y(t, q(t, x)) q_{x}^{\frac{\beta}{N}}(t, x)=y_{0}(x) e^{-\lambda t},
$$

which exactly yields Eq. (2.7):

$$
\begin{aligned}
& e^{-\lambda t}\left\|y_{0}\right\|_{L^{\frac{N}{\beta}}}^{\frac{N}{\beta}}=\left\|y(t, q(t, \cdot)) q_{x}^{\frac{\beta}{N}}(t, \cdot)\right\|_{L^{\frac{N}{\beta}}}^{\frac{N}{\beta}} \\
= & \int_{\mathbb{R}}|y(t, q(t, x))|^{\frac{N}{\beta}} q_{x}(t, x) d x \\
= & \left.\int_{\mathbb{R}}|y(t, z)|^{\frac{N}{\beta}} d z \quad \text { by setting } q(t, x)=z\right) .
\end{aligned}
$$

Apparently, letting $N=2 \beta$ leads to Eq. (2.8). 


\section{Global existence}

In this section, we provide two global existence results for strong solutions to Eq. (1.2).

Theorem 3.1. Assume $u_{0} \in H^{s}(\mathbb{R}), s \geq 3$, and $y_{0}=u_{0}-u_{0, x x}$ satisfies $y_{0}(x) \neq 0$ a.e. $x \in \mathbb{R}$ and $\left\|y_{0}\right\|_{L^{2}} \leq\left(\frac{2^{\frac{\bar{N}}{}+1} \lambda}{|N-2 \beta|}\right)^{\frac{1}{N}}$, where $\beta \neq \frac{N}{2}$. Then the solution $u(t, x)$ to Eq. (1.2) globally exists.

Proof. Multiplying both sides of Eq. (2.4) by $e^{2 \lambda t}$ yields

$$
\frac{d}{d t}\left(e^{2 \lambda t} \int_{\mathbb{R}} y^{2} d x\right)=\left(1-\frac{2 \beta}{N}\right) e^{2 \lambda t} \int_{\mathbb{R}} y^{2} N u^{N-1} u_{x} d x .
$$

Employing $G=\frac{1}{2} e^{-|x|}, G * y=u$, and $G_{x} * y=u_{x}$, and using the Young's inequality, we have

$$
\|u\|_{L^{\infty}} \leq\|G\|_{L^{2}}\|y\|_{L^{2}}=\frac{1}{2}\|y\|_{L^{2}},
$$

and

$$
\left\|u_{x}\right\|_{L^{\infty}} \leq\left\|G_{x}\right\|_{L^{2}}\|y\|_{L^{2}}=\frac{1}{2}\|y\|_{L^{2}}
$$

which implies

$$
\frac{d}{d t}\left(e^{2 \lambda t} \int_{\mathbb{R}} y^{2} d x\right) \leq|N-2 \beta| e^{2 \lambda t}\left(\frac{1}{2}\|y\|_{L^{2}}\right)^{N} \int_{\mathbb{R}} y^{2} d x .
$$

Thus, we obtain

$$
\begin{array}{r}
\frac{d}{d t}\left(e^{2 \lambda t} \int_{\mathbb{R}} y^{2} d x\right) \leq|N-2 \beta| e^{2 \lambda t}\left(\frac{1}{2}\|y\|_{L^{2}}\right)^{N}\|y\|_{L^{2}}^{2} \\
=\frac{|N-2 \beta|}{2^{N}} e^{-N \lambda t}\left(e^{2 \lambda t} \int_{\mathbb{R}} y^{2} d x\right)^{\frac{N+2}{2}} .
\end{array}
$$

Let $f(t):=e^{2 \lambda t} \int_{\mathbb{R}} y^{2} d x$. We claim that if $y_{0}(x) \neq 0$ a.e. $x \in \mathbb{R}$, then $f(t)>0$ for all $t \in[0, T)$. Apparently from Eq. (2.6), if $y_{0}(x) \neq 0$ a.e. $x \in \mathbb{R}$, then $y(t, q(t, x)) \neq 0$ a.e. $x \in \mathbb{R}$. Therefore, we have

$$
\int_{\mathbb{R}} y^{2}(t, z) d z=\int_{\mathbb{R}} y^{2}(t, q(t, x)) q_{x}(t, x) d x>0,
$$

which implies $f(t)>0$. Solving (3.3) for $f(t)$ leads to

$$
\frac{d}{d t}\left(f(t)^{-\frac{N}{2}}\right) \geq-\frac{N}{2} \frac{|N-2 \beta|}{2^{N}} e^{-N \lambda t},
$$


which can be integrated with respect to $t$ to give

$$
\begin{array}{r}
f(t)^{-\frac{N}{2}}-f(0)^{-\frac{N}{2}} \geq \\
-\frac{N}{2} \frac{|N-2 \beta|}{2^{N}} \int_{0}^{t} e^{-N \lambda s} d s \\
=\frac{N|N-2 \beta|}{2^{N+1}} \frac{e^{-N \lambda t}-1}{N \lambda},
\end{array}
$$

that is,

$$
f(t)^{-\frac{N}{2}} \geq f(0)^{-\frac{N}{2}}-\frac{|N-2 \beta|}{2^{N+1} \lambda}
$$

Due to $\beta \neq \frac{N}{2}$ and $y_{0}(x) \neq 0$ a.e. $x \in \mathbb{R}$, therefore if $f(0)^{-\frac{N}{2}}-\frac{|N-2 \beta|}{2^{N+1} \lambda}>0$, then we have $\left\|y_{0}\right\|_{L^{2}} \leq\left(\frac{2^{N+1} \lambda}{|N-2 \beta|}\right)^{\frac{1}{N}}$ and

$$
\left[f(0)^{-\frac{N}{2}}-\frac{|N-2 \beta|}{2^{N+1} \lambda}\right]^{-1} \geq f(t)^{\frac{N}{2}}
$$

i.e.

$$
\left[\left(\int_{\mathbb{R}} y_{0}^{2} d x\right)^{-\frac{N}{2}}-\frac{|N-2 \beta|}{2^{N+1} \lambda}\right]^{-1} \geq\left(e^{2 \lambda t} \int_{\mathbb{R}} y^{2} d x\right)^{\frac{N}{2}} .
$$

Therefore, we obtain

$$
\|y\|_{L^{2}}^{N} \leq\left(e^{-N \lambda t}\right)\left[\left\|y_{0}\right\|_{L^{2}}^{-N}-\frac{|N-2 \beta|}{2^{N+1} \lambda}\right]^{-1} .
$$

By (3.1), (3.2) and (3.4) one may readily see that $u$ and $u_{x}$ are bounded:

$$
\|u\|_{L^{\infty}},\left\|u_{x}\right\|_{L^{\infty}} \leq \frac{1}{2}\|y\|_{L^{2}} \leq \frac{1}{2}\left(e^{-\lambda t}\right)\left[\left\|y_{0}\right\|_{L^{2}}^{-N}-\frac{|N-2 \beta|}{2^{N+1} \lambda}\right]^{-\frac{1}{N}},
$$

which guarantee the global existence of the solution $u(t, x)$ to Eq. (1.2).

Remark 3.1. Theorem 3.1 includes the result of Theorem 1 in [37], since the $C H$ equation is a special case of Eq. (1.1) with $N=1$ and $\beta=2$.

Remark 3.2. Theorem 3.1 shows that the dispersion term $k y_{x}$ does not affect the global existence of strong solution to Eq. (1.2), but the dissipation term $\lambda y$ does.

Let us now discuss the special case either $\beta=\frac{N}{2}$ or $\beta=N+1$. To do so, we shall use a family of diffeomorphisms on a line to study the global existence of strong solutions to Eq.(1.2).

Theorem 3.2. Assume $u_{0} \in H^{s}(\mathbb{R}), s \geq 3$, is a given initial data such that the associated potential $y_{0}=u_{0}-u_{0, x x}$ does not change sign. Then when $\beta=N+1$ or $\beta=\frac{N}{2}$ the corresponding solution $u(t, x)$ to Eq. (1.2) globally exists. 
Proof. Without loss of generality, let us assume $y_{0} \geq 0$. Lemma 2.8 tells us that $y(t, x) \geq 0$ for $x \in \mathbb{R}, t \geq 0$. Since $u=G * y$ and $G \geq 0$, one may immediately see $u(t, x) \geq 0$ for all time $t \geq 0$. Obviously with the aid of $u=\frac{1}{2} e^{-|x|} * y$, we may express $u$ and $u_{x}$ as follows:

$$
\begin{array}{r}
u(t, x)=\frac{e^{-x}}{2} \int_{-\infty}^{x} e^{z} y(t, z) d z+\frac{e^{x}}{2} \int_{x}^{\infty} e^{-z} y(t, z) d z, \\
u_{x}(t, x)=-\frac{e^{-x}}{2} \int_{-\infty}^{x} e^{z} y(t, z) d z+\frac{e^{x}}{2} \int_{x}^{\infty} e^{-z} y(t, z) d z .
\end{array}
$$

Therefore, we have

$$
\begin{array}{r}
u(t, x)+u_{x}(t, x)=e^{x} \int_{x}^{\infty} e^{z} y(t, z) d z \geq 0, \\
u(t, x)-u_{x}(t, x)=e^{-x} \int_{-\infty}^{x} e^{z} y(t, z) d z \geq 0 .
\end{array}
$$

i.e. $\left|u_{x}\right| \leq u$.

When $\beta=N+1$, Lemma 2.6 yields the following inequalities

$$
\left|u_{x}\right| \leq u \leq\|u\|_{L^{\infty}} \leq \frac{1}{\sqrt{2}}\|u\|_{H^{1}} \leq \frac{1}{\sqrt{2}}\left\|u_{0}\right\|_{H^{1}} .
$$

When $N=2 \beta$, Eq. (2.8) leads to

$$
\left|u_{x}\right| \leq u \leq\|u\|_{L^{\infty}} \leq \frac{1}{\sqrt{2}}\|u\|_{H^{1}} \leq \frac{1}{\sqrt{2}}\|u\|_{H^{2}} \leq \frac{1}{\sqrt{2}}\|y\|_{L^{2}} \leq \frac{1}{\sqrt{2}}\left\|y_{0}\right\|_{L^{2}},
$$

where we used the following relations

$$
\begin{aligned}
& \|u\|_{H^{2}}^{2} \leq\|y\|_{L^{2}}^{2}=\int_{\mathbb{R}}\left(u-u_{x x}\right)^{2} d x=\int_{\mathbb{R}}\left(u^{2}-2 u u_{x x}+u_{x x}^{2}\right) d x \\
& =\int_{\mathbb{R}}\left(u^{2}+2 u_{x}^{2}+u_{x x}^{2}\right) d x \leq 2\|u\|_{H^{2}}^{2} .
\end{aligned}
$$

So, (3.8) and (3.9) combining with Theorem 2.3 complete the proof.

Remark 3.3. Theorem 3.2 covers Theorem 1.4 in [29].

Remark 3.4. Comparing Theorem 3.1 with Theorem 3.2, we see a very interesting physical phenomenon. In the case of $\beta \neq \frac{N}{2}$, the size of the initial potential $y_{0}$ in $L^{2}$ space impacts the lifespan, namely, the small initial potential guarantees the global existence. However, in the case of $\beta=N+1$ or $\beta=\frac{N}{2}$, neither the smoothness nor the size of the initial data affects the lifespan, but the sign of the initial potential $y_{0}$ does. 


\section{Propagation speed}

In this section, we shall investigate impact of the dispersion coefficient $k$ and the dissipative parameter $\lambda$ on the propagation speed of solutions to Eq. (1.2).

Theorem 4.1. Assume that for some $T>0$ and $s \geq 3, u \in C\left([0, T] ; H^{s}(\mathbb{R})\right)$ is a strong solution of the initial value problem associated with Eq. (1.2). If the initial data $u_{0}(x)$ is compactly supported on $[a, b]$, and if either $\beta=N$, where $N$ is a positive odd number or $N=1,0 \leq \beta \leq 3$, then for any $t \in$ $[0, T]$, we have $u(t, x)=\frac{1}{2} E_{+}(t) e^{-x}$, for $x \geq q(t, b)$, and $u(x, t)=\frac{1}{2} E_{-}(t) e^{x}$, for $x \leq q(t, a)$, where $E_{+}(t)$ and $E_{-}(t)$ stand for continuous non-vanishing functions with $E_{+}(t)>0$ and $E_{-}(t)<0$ for $t \in[0, T]$.

Proof. If $u_{0}$ is initially compact-supported on the closed interval $[a, b]$, then so is $y_{0}$. It follows from Lemma 2.8 that $y(t, \cdot)$ is compactly supported within the interval $[q(t, a), q(t, b)]$.

Let us define the following two functions

$$
E_{+}(t)=\int_{q(t, a)}^{q(t, b)} e^{z} y(t, z) d z, \quad E_{-}(t)=\int_{q(t, a)}^{q(t, b)} e^{-z} y(t, z) d z .
$$

Then by (3.5)-(3.7) we have

$$
\begin{gathered}
u(t, x)=\frac{e^{-x}}{2} E_{+}(t), \quad x>q(t, b), \\
u(t, x)=\frac{e^{x}}{2} E_{-}(t), \quad x<q(t, a),
\end{gathered}
$$

which generate the following derivative formulas

$$
\begin{gathered}
\frac{e^{-x}}{2} E_{+}(t)=u(t, x)=-u_{x}(t, x)=u_{x x}(t, x), \quad x>q(t, b), \\
\frac{e^{x}}{2} E_{-}(t)=u(t, x)=u_{x}(t, x)=u_{x x}(t, x), \quad x<q(t, a) .
\end{gathered}
$$

Since $u(0, \cdot)$ is compactly supported on the interval $[a, b]$, this immediately gives us $E_{+}(0)=E_{-}(0)=0$.

Noticing that $y(t, \cdot)$ is compactly supported on the interval $[q(t, a), q(t, b)]$, for each fixed $t$ we have

$$
\frac{d E_{+}(t)}{d t}=\int_{q(t, a)}^{q(t, b)} e^{z} y_{t}(t, z) d z=\int_{-\infty}^{\infty} e^{z} y_{t}(t, z) d z
$$


which yields

$$
\begin{aligned}
& \frac{d E_{+}(t)}{d t}=\int_{q(t, a)}^{q(t, b)} e^{z} y_{t}(t, z) d z \\
& =\int_{-\infty}^{\infty} e^{z} y_{t}(t, z) d z \\
& =-\int_{-\infty}^{\infty}\left(-y_{x} u^{N}-\frac{\beta}{N} y\left(u^{N}\right)_{x}-k y_{x}-\lambda y\right) e^{z} d z \\
& =\frac{\beta}{N+1} \int_{-\infty}^{\infty} e^{z} u^{N+1} d z+\frac{3 N-\beta}{2} \int_{-\infty}^{\infty} e^{z} u^{N-1} u_{z}^{2} d z \\
& \quad+\frac{(N-\beta)(N-1)}{2} \int_{-\infty}^{\infty} e^{z} u^{N-2} u_{z}^{3} d z-(\lambda-k) \int_{-\infty}^{\infty} e^{z} y d z
\end{aligned}
$$

Let

$$
\begin{array}{r}
F:=\frac{\beta}{N+1} \int_{-\infty}^{\infty} e^{z} u^{N+1} d z+\frac{3 N-\beta}{2} \int_{-\infty}^{\infty} e^{z} u^{N-1} u_{z}^{2} d z \\
+\frac{(N-\beta)(N-1)}{2} \int_{-\infty}^{\infty} e^{z} u^{N-2} u_{z}^{3} d z .
\end{array}
$$

If either $\beta=N$ ( $N$ is a positive odd number) or $N=1,0 \leq \beta \leq 3$, then

$$
\frac{d}{d t} E_{+}(t)+(\lambda-k) E(t)=F \geq 0,
$$

which implies

$$
\frac{d}{d t}\left(e^{(\lambda-k) t} E_{+}(t)\right) \geq 0
$$

that is

$$
e^{(\lambda-k) t} E_{+}(t) \geq 0
$$

Therefore, $E_{+}(t) \geq 0$.

Adopting the similar procedure for $E_{-}(t)$ as above, we can arrive at

$$
\frac{d E_{-}(t)}{d t}=\int_{q(t, a)}^{q(t, b)} e^{-z} y_{t}(t, z) d z=-F+(-\lambda-k) \int_{-\infty}^{\infty} e^{-z} y d z .
$$

So, $E_{-}(t) \leq 0$. This completes the proof of Theorem 4.1.

Remark 4.1. From the proof of Theorem 4.1, we see that there are significant differences between the cases $k, \lambda \neq 0$ and $k, \lambda=0$. More precisely, the first order derivatives of the functions $E_{+}(t)$ and $E_{-}(t)$ in the case $k, \lambda \neq 0$, in general, can change their signs. While Theorem 4.1 in [29] with $k=\lambda=0$ showed that $E_{+}(t)$ and $E_{-}(t)$ are strictly decreasing for $t \in[0, T)$. 
Acknowledgments This work was supported by NSFC (Nos. 11401223, 11171295, and 61328103), Guangdong NSF (No. 2015A030313424) and China Scholarship Council. The first author would like to thank Professor Zhijun Qiao for his kind hospitality and encouragement during her visit in the University of Texas - Rio Grande Valley, and the second author thanks the U.S. Department of Education GAANN project (P200A120256) for supporting the UTPA mathematics graduate program.

\section{References}

[1] A. Bressan and A. Constantin, Global conservative solutions of the Camassa-Holm equation, Arch. Rat. Mech. Anal., 183 (2007), 215-239.

[2] R. Camassa and D. Holm, An integrable shallow water equation with peaked solitons, Phys. Rev. Letters, 71 (1993), 1661-1664.

[3] R. Camassa, D. Holm and J. Hyman, A new integrable shallow water equation, Adv. Appl. Mech., 31 (1994), 1-33.

[4] G. Coclite and K. Karlsen, On the well-posedness of the DegasperisProcesi equation, J. Funct. Anal., 233 (2006), 60-91.

[5] A. Constantin, The Hamiltonian structure of the Camassa-Holm equation, Exposition. Math., 15 (1997), 53-85.

[6] A. Constantin, The Cauchy problem for the periodic Camassa-Holm equation, J. Differential Equations, 141 (1997), 218-235.

[7] A. Constantin, On the blow-up of solutions of a periodic shallow water equation, J. Nonlinear Sci., 10 (2000), 391-399.

[8] A. Constantin, Existence of permanent and breaking waves for a shallow water equation: a geometric approach, Ann. Inst. Fourier, Grenoble, 50 (2000), 321-362.

[9] A. Constantin, On the scattering problem for the Camassa-Holm equation, Proc. R. Soc. London A, 457 (2001), 953-970.

[10] A. Constantin, The trajectories of particles in Stokes waves, Invent. Math., 166 (2006), 523-535.

[11] A. Constantin and J. Escher, Wave breaking for nonlinear nonlocal shallow water equations, Acta Mathematica, 181 (1998), 229-243.

[12] A. Constantin and J. Escher, Well-posedness, global existence, and blowup phenomena for a periodic quasi-linear hyperbolic equation, Comm. Pure Appl. Math., 51 (1998), 475-504. 
[13] A. Constantin and J. Escher, On the structure of a family of quasilinear equations arising in a shallow water theory, Math. Ann., 312 (1998), $403-416$.

[14] A. Constantin and J. Escher, Particle trajectories in solitary water waves, Bull. Amer. Math. Soc., 44 (2007), 423-431.

[15] A. Constantin and L. Molinet, The initial value problem for a generalized Boussinesq equation, Differential and Integral equations 15 (2002) 1061-1072.

[16] A. Degasperis and M. Procesi, Asymptotic integrability symmetry and perturbation theory, (Rome, 1998), World Sci. Publ., (1999), 23-37.

[17] A. Degasperis, D. Holm and A. Hone, A new integrable equation with peakon solution, Theoret. and Math. Phys, 133 (2002) 1463-1474.

[18] Q. Hu and Z. Yin, Blowup and blowup rate of solutions to a weakly dissipative periodic rod equation, J.Math. Phys., 50083503 (2009), $1-16$.

[19] Q. Hu, Global existence and blow-up phenomena for a weakly dissipative periodic 2-component Camassa-Holm system, J. Math. Phys., 52 103701 (2011), 1-13.

[20] J. Escher, Y. Liu and Z. Yin, Global weak solutions and blow-up structure for the Degasperis-Procesi equation, J. Funct. Anal., 241 (2006), $257-485$.

[21] J. Escher, Y. Liu and Z. Yin, Shock waves and blow-up phenomena for the periodic Degasperis-Procesi equation, Indiana Univ. Math. J., 56 (2007), 87-117.

[22] A. Fokas, On a class of physically important integrable equations, Physica D 87 (1995), 145-150.

[23] A. Fokas and B. Fuchssteiner, Symplectic structures, their Bäcklund transformation and hereditary symmetries, Physica D, 4 (1981), 4766.

[24] J. Ghidaglia, Weakly damped forced Korteweg-de Vries equations behave as a finite dimensional dynamical system in the long time, $J$. Differential Equations, 74 (1988), 369-390.

[25] A. Himonas and C. Holliman, The Cauchy problem for a generalized Camassa-Holm equation, Advances in Differential Equations, 19 (2013), 161-200. 
[26] A. Himonas and C. Holliman, On well-posedness of the DegasperisProcesi equation, Discrete Contin. Dyn. Syst., 31 (2011), 469-488.

[27] A. Himonas, C. Holliman and K. Grayshan, Norm inflation and illposedness for the Degasperis-Procesi equation, Comm. Partial Differential Equations 39 (2014) 2198-2215.

[28] A. Himonas and C. Holliman, The Cauchy problem for the Novikov equation, Nonlinearity, 25 (2012), 449-479.

[29] A. Himonas and R. Thompson, Persistence properties and unique continuation for a generzlized Camassa-Holm Equation, J. Math. Phys., 55 (2014), 091503.

[30] A. Hone and J. Wang, Integrable peakon equations with cubic nonlinearity, J. Phys. A: Math. Theor. 41 (2008), 372002-372010.

[31] T. Kato, Quasi-linear equations of evolution, with applications to partial differential equations, in: Spectral Theory and Differential Equations, Lecture Notes in Math., Springer Verlag, Berlin, 448 (1975), $25-70$.

[32] T. Kato and G. Ponce, Commutator estimates and Navier-Stokes equations, Comm. Pure Appl. Math., 41 (1988), 203-208.

[33] Y. Li and P. Olver, Well-posedness and blow-up solutions for an integrable nonlinearly dispersive model wave equation, J. Differential Equations, 162 (2000), 27-63.

[34] Y. Li and P. Olver, Well-posedness and blow-up solutions for phenomona for the Degasperis-Procesi equation, Comm. Math. Phys., 267 (2006), 801-820.

[35] H. McKean, Breakdown of a shallow water equation, Asian J. Math., 2(4) (1998) 867-874.

[36] V. Novikov, Generalizations of the Camassa-Holm equation, J. Phys. A, 42 (2009), 342002, 14 pages.

[37] E. Novruzova and A. Hagverdiyevb, On the behavior of the solution of the dissipative Camassa-Holm equation with the arbitrary dispersion coefficient J. Differential Equations, 257 (2014), 4525-4541.

[38] P. Olver and P. Rosenau, Tri-Hamiltonian duality between solitons and solitary-wave solutions having compact support, Phys. Rev. E, 53 (1996), 1900-1906.

[39] E. Ott and R. Sudan, Damping of solitary waves, Phys. Fluids, 13 (1970), 1432-1434. 
[40] Z. Qiao, The Camassa-Holm hierarchy, related $N$-dimensional integrable systems and algebro-geometric solution on a symplectic submanifold, Commun. Math. Phys. 239 (2003) $309-341$.

[41] Z. Qiao, Integrable hierarchy (the DP hierarchy), $3 \times 3$ constrained systems, and parametric and stationary solutions, Acta Applicandae Mathematicae 83 (2004), 199-220.

[42] Z. Qiao, A new integrable equation with cuspons and W/M-shape-peaks solitons, J. Math. Phys. 47 (2006), $112701-112710$.

[43] G. Rodriguez-Blanco, On the Cauchy problem for the Camassa-Holm equation, Nonlinear Anal., 46 (2001), 309-327.

[44] J. Toland, Stokes waves, Topol. Methods Nonlinear Anal., 7 (1996), $1-48$.

[45] F. Tiğlay, The periodic Cauchy problem for Novikov's equation, Int. Math. Res. Not., (2010) rnq267, 1-16.

[46] S. Wu and Z. Yin, Blow-up, blow-up rate and decay of the solution of the weakly dissipative Camassa-Holm equation, J. Math. Phys., 47, 013504 (2006), 1-12.

[47] S. Wu and Z. Yin, Blow-up and decay of the solution of the weakly dissipative Degasperis-Procesi equation, SIAM J. Math. Anal., 40 (2008), 475-490.

[48] X. Wu and Z. Yin, Well-posedness and global existence for the Novikov equation, Ann. Sc. Norm. Sup. Pisa CI. Sci., 5 (2012), 707-727.

[49] X. Wu and Z. Yin, Global weak solutions for the Novikov equation, J. Phys. A: Math. Theor., 44 (2011), 055202 1-17.

[50] Z. Xin and P. Zhang, On the weak solutions to a shallow water equation, Comm. Pure Appl. Math., 53 (2000), 1411-1433.

[51] Z. Yin, Global existence for a new periodic integrable equation, J. Math. Anal. Appl., 283 (2003), 129-139.

[52] Z. Yin, On the Cauchy problem for an integrable equation with peakon solutions, Illinois J. Math., 47 (2003), 649-666.

[53] S. Zhou and C. Mu, The properties of solutions for a generalized bfamily equation with peakons, J Nonlinear Sci, 23 (2013), 863-889.

[54] S. Zhou, C. Mu and L. Wang, Well-posedness, blow-up phenomena and global existence for the generalized b-equation with higher-order nonlinearities and weak diddipation, Discrete Contin. Dyn. Syst. Ser. A, 34 (2014), 843-867. 